\title{
Algunas características familiares y de la escuela que contribuyen a la etiología de la tensión emocional
}

\author{
Gladys Jadue ${ }^{1}$ \\ Universidad Austral de Chile
}

El artículo analiza algunas particularidades familiares y de la escuela que contribuyen a la aparición de estados ansiosos en los alumnos, que afectan tanto su desarrollo psicosocial como su rendimiento escolar. Enfatiza las características de los estados ansiosos que pueden ser provocados o exacerbados por la escuela y que junto con algunas peculiaridades familiares ansiogénicas pueden aumentar el número de niños en riesgo de bajo rendimiento y de fracaso escolar. Sostiene que tanto a través de una comunicación intersistémica eficiente familia-escuela como en el desarrollo de los Objetivos Fundamentales Transversales contenidos en la Reforma educacional chilena, es posible aminorar la sintomatología ansiosa, con el consecuente beneficio tanto para el desarrollo personalafectivo, como en el rendimiento escolar de los alumnos.

Palabras claves: tensión emocional, ansiedad, rendimiento escolar, características familiares y escolares

\section{Some family and school characteristics affecting the etiology or emotional disturbance}

This paper analyses and describes some important characteristics of families and the school affecting the students both in their psychosocial development and their school performance. It also emphasizes the characteristics of anxiety that can be trigger by the school and that together with some family anxiety peculiarities can increase the number of children who are at risk of having a low school performance and school failure. This paper suggest that in order to improve student's psychosocial development together with school performance, it is important to have an efficient intersystemic communication between the family and the school. It is also important to apply reliable strategies, as those contained in the Chilean Educational Reform so as to achieve these goals.

Key words: emotional disturbance, anxiety, school performance, family and school characteristics.

1 Doctora en psicología educacional de la Universidad de Kentucky (EUA). Chilena. Psicóloga clínica de la Universidad de Paris V Sorbona (Francia). Psicóloga de la Universidad de Chile. Profesora Titular y Directora del Instituto de Filosofía y de Estudios Educacionales de la Universidad Austral de Chile - Valdivia. Correo electrónico: jmoraga@uach.cl; gjadue@valdivia.uca.uach.cl. 

En las culturas individualistas competitivas las personas disfrutan de mayor libertad personal, tienen más orgullo de sus logros, disfrutan de más privacidad y se sienten más libres de circular y de elegir sus propios estilos de vida. Sin embargo, el costo personal es, entre otros, mayor vulnerabilidad ante los síntomas y las enfermedades relacionadas con la tensión emocional (Triandis, Bontemps, Villareal, Assai y Lucca, 1988).

La ansiedad en el niño, uno de los síntomas más comunes de la tensión emocional, es una importante pero desatendida área de investigación. Cada vez más niños escolares presentan problemas emocionales en la escuela (Doll y Lyon, 1998). Estudios epidemiológicos indican que los desórdenes ansiosos durante el desarrollo afectan aproximadamente al $9 \%$ de los niños (Berstein y Borchard, 1991) y entre el 13 y el $17 \%$ de los jóvenes (Kashani y Overvaschel, 1990). Otras referencias indican que entre el 10 y el $20 \%$ de los escolares se ven afectados por problemas relacionados con la ansiedad (Barret, 1998). Muchas de las demandas de atención en los consultorios de salud infanto-juveniles en Chile, tienen relación con problemas de aprendizaje en la escuela. La mayoría de los estados depresivo-ansiosos de esta etapa se relacionan con el bajo rendimiento y el fracaso escolar.

Sin embargo, la prevalencia de los trastornos ansiosos es incierta, ya que a menudo los niños a quienes se les diagnostica algún trastorno ansioso, suelen responder, además, a criterios de una o mas perturbaciones adicionales (Wicks-Nelson e Israel, 1997). Existen también, combinaciones de problemas conductuales que coinciden con concepciones de trastornos ansiosos en niños y adolescentes (Achembach, 1993). Asimismo, cada niño y cada adolescente muestran distintos síntomas ansiosos y con diversa intensidad. Pueden variar también tanto en su 
persistencia como en el período en el que se presentan, obedeciendo a tres sistemas de respuestas: motoras, fisiológicas y subjetivas.

\section{Escuela Ansiogénica}

Una circunstancia ambiental que contribuye al desarrollo de la ansiedad proviene del sistema escolar donde circunstancias no fortuitas, motivadas por una situación de verdadero riesgo o amenaza, afectan al niño aún antes de que éste se incorpore a la escuela. El actual sistema de clasificación para ingresar a algunos establecimientos educacionales en Chile, involucra niños muy pequeños que se ven obligados a competir para ser aceptados como alumnos. Se les administran un conjunto de pruebas psicométricas que clasifican al postulante dentro de un ranking establecido por el resultado obtenido en este verdadero examen de admisión , quedando fuera del establecimiento, niños normales que no presentan ninguna alteración ni retraso. Esta situación provoca ansiedad en el niño, como asimismo una desilusión, estados depresivos y/o ansiosos en los padres que tenían expectativas educacionales para sus hijos referidas a ese colegio, tanto por la calidad de la educación que otorga sino también por lo que significa socialmente ser alumno de dicho establecimiento. El miedo y la angustia resultan entonces una respuesta normal frente a una escuela deshumanizada. La ansiedad surge ante una situación que no permite tomar medidas defensivas, frente a una amenaza al status y al rol . Asimismo, los padres se ven sorprendidos cuando su hijo aparentemente normal, es considerado desventajoso (Bender, 1996). En efecto, los estudiantes raramente son diagnosticados con problemas de aprendizaje hasta que han mostrado bajo rendimiento escolar. El procedimiento de clasificación ha mostrado ser destructivo para los niños y su continua ejecución simplemente revela la tendencia de las escuelas a excluir a aquellos niños que eventualmente puedan presentar problemas de la conducta y del aprendizaje escolar.

Una vez que ingresan al sistema escolar, cada día en la escuela los niños se esfuerzan para establecer y mantener relaciones interpersonales, 
esforzándose para desarrollar identidades sociales y un sentido de pertenencia. Observan y adquieren modelos de conducta que imitan de los otros y son gratificados por comportarse de una manera aceptable para sus compañeros y para sus profesores. Generalmente aquellos niños que tienen éxito en el contexto social, son los más exitosos como estudiantes.

La evidencia empírica indica que la ansiedad en la niñez tiene implicancias negativas en el ámbito psicosocial ya que está asociada con significativos deterioros en sus relaciones con sus pares y en su competencia social (Cobhan, Dadds y Spence, 1998). Si la ansiedad es intensa, el niño muestra excesiva sensibilidad y temores, incluyendo temor a la escuela, verguienza, timidez, sensaciones importantes de insuficiencia y síntomas somáticos. Los niños intensamente ansiosos padecen de una extraordinaria tendencia a preocuparse, están excesivamente preocupados por su competencia y rendimiento escolar exteriorizando intranquilidad, trastornos del sueño, dolores de estómago, etc. Asimismo, tienen temor de no gustarle a los demás niños, sufren la sensación de que sus compañeros se ríen de ellos y se muestran aprensivos frente a actividades que otros niños realizan con entusiasmo. El alumno ansioso a menudo presenta problemas en su socialización porque su dependencia del adulto y sus propias aprensiones los hacen aparecer muy infantiles ante sus compañeros.

Un estado ansioso elevado provoca que el niño se altere fácilmente por experiencias de la vida cotidiana y especialmente ante la tarea escolar ya que cuando sufre de elevada ansiedad, muestra un comportamiento y un rendimiento escolar diferente al resto de sus compañeros. Exhibe desasosiego y un miedo exagerado y constante a actuar de una manera vergonzante o sorprendente en situaciones o actividades donde se reúnen varias personas. Para niños entre 8 y 12 años de edad los miedos sociales más frecuentes están relacionados con situaciones en las que hay que hablar y/o leer en voz alta, hablar con personas que representen autoridad, así como también relacionarse con sus pares (Beidel y Randall, 1994). La ansiedad social entre estas edades pueden agruparse de acuerdo a tres 
signos (La Greca y Stone, 1993): miedo a las evaluaciones negativas de sus compañeros; evitación del contacto social; y angustia frente a situaciones nuevas. Asimismo, la ansiedad social está ligada a la poca aceptación de parte de los compañeros de curso y/o amigos y de una autoestima deteriorada.

Cogniciones específicas pueden desarrollar la percepción de situaciones como algo hostil y amenazador; pueden hacer que el niño corra el riesgo de desarrollar o mantener trastornos ansiosos (Bell-Dollan, 1995). Sin embargo, el niño puede tener dificultades para verbalizar y comunicar sus emociones y sentimientos, lo que supone una dificultad para realizar la evaluación correspondiente.

El profesor capaz de percibir el sentimiento de impotencia en el alumno, puede actuar para prevenir la ansiedad y facilitar el control. Los alumnos que presentan sentimientos de impotencia, generalmente muestran una hostilidad sistemática hacia un tema. Probablemente no se observe con claridad la naturaleza del problema del alumno, pero es evidente que enfrenta una dificultad y no se siente en condiciones de resolverla. Es posible además que el alumno presente actitudes de desconcierto o de bloqueo frente a determinados contenidos, a pesar de dársele explicaciones adecuadas. Este bloqueo o el no entender puede manifestarse como falta de participación en clases, poca atención y falta en el cumplimiento de las tareas que debe hacer en casa. Este tipo de alumno muestra dependencia de su profesor y/o de sus compañeros para obtener las respuestas a preguntas que el mismo podría resolver con un poco de reflexión y trabajo personal; de tal manera que es un alumno que rinde bien en un grupo bajo supervisión, pero que tiene dificultades o no es capaz de iniciar o continuar por si solo una actividad académica.

Altos niveles de ansiedad, reducen la eficiencia en el aprendizaje, ya que disminuyen la atención, la concentración y la retención con el consecuente deterioro en el rendimiento escolar. Los muy ansiosos tienen dificultades para poner atención, se distraen con facilidad. Utilizan pocas de las claves que se otorgan en las tareas intelectuales. A medida en que 
van procesando la información, no organizan ni elaboran adecuadamente los materiales y tienden a ser poco flexibles para adaptarse a los procesos de aprendizaje (Newcomer, 1993). Aquellos alumnos con una inteligencia promedio, sufren mayormente de dificultades académicas, ya que los mejores dotados intelectualmente pueden compensar la ansiedad (Spielberger, 1985).

Por su misma naturaleza, la ansiedad depende del concepto que el alumno tiene de las demandas que se le formulan en relación con la capacidad de comprensión y de control que encuentra en si mismo. El niño enfrenta constantemente situaciones académicas cuyas exigencias debe comparar con sus propios medios. Cuando su evaluación de las demandas de la escuela lo llevan a concluir que son más de lo que el puede rendir, afronta una situación de peligro, de humillación, a veces suficientes para justificar el rechazo a la escuela (Jackson y Frick, 1998).

La frustración es uno de los factores que pueden llevar a la conducta agresiva, ya que fomenta la hostilidad entre los alumnos. La frustración es una experiencia desagradable y aversiva que conduce a la agresividad debido a una relación básica entre afectos negativos y comportamientos agresivos, relación confirmada empíricamente (da Gloria, Pahlavan, Duda y Bonnet, 1994). La competencia entre los niños genera gran cantidad de ira y agresión. La rivalidad genera hostilidad y está vinculada con la agresión, de tal manera que cuando los niños compiten por rendimiento académico la violencia intraescolar aumenta (Turner, Fenn y Cole , 1981). La estabilidad personal y emocional está ligada con la tolerancia a la frustración. En general, el alumno que tiene antecedentes de inestabilidad emocional tiene menores posibilidades de resistir los efectos de la frustración que aquellos emocionalmente estables. Por lo tanto el niño que sufre ansiedad está en alto riesgo de trastornos conductuales asociados a su inestabilidad emocional.

Asimismo, la ansiedad intensa puede provocar un "déficit cognitivo" (Zillmann, 1984); es decir, menor capacidad de formular planes de acción racionales y/o de evaluar las eventuales consecuencias de distintos 
comportamientos impulsivos, lo que puede conllevar un aumento de los impulsos agresivos.

\section{Tensión emocional y rendimiento escolar}

Los problemas emocionales, generalmente son citados tanto como complicaciones secundarias de los trastornos del aprendizaje como en la etiología de los mismos. Estudiantes con leves trastornos del aprendizaje y de la conducta escolar, muestran deficiencias en cogniciones, memoria, lenguaje, atención, relaciones sociales y desarrollo emocional (Patton, Blackbourn y Fad, 1996). En niños atendidos en grupos diferenciales diagnosticados dentro de la categoría "problemas de aprendizaje" entendida como retraso en el rendimiento escolar que no reúne los requisitos para constituir un "trastorno", pero que puede darse concomitantemente con este, un $40.1 \%$ de los niños se asignó al subgrupo "perturbaciones emocionales" (Bermeosolo y Pinto, 1996). No es raro que un niño o un adolescente responda con frecuencia a los criterios de más de un trastorno, y que en él se sumen una serie de situaciones de riesgo compartidas.

Frente a la confluencia de distintos factores etiológicos y complicaciones secundarias que emergen en el niño con problemas conductuales, sociales, emocionales y del aprendizaje escolar, existen diferentes designaciones diagnósticas: niño en riesgo de bajo rendimiento o de fracaso escolar, niño en desventaja, niño deprivado sociocultural y económicamente, niño con retraso maduracional, niño con síndrome de déficit atencional, niño con trastorno del desarrollo, niño con problemas del aprendizaje, niño con trastorno del aprendizaje, niño con trastorno emocional, niño ansioso, niño hiperansioso, niño retraído, niño tímido, con trastorno depresivo, con trastorno conductual, etc.

Se trata, en general, de menores que padecen de dificultades del aprendizaje escolar y de la conducta, cuya etiología es de origen orgánico, psíquico o no precisada claramente, que se diferencian entre sí, a veces 
muy sutilmente, por algunos signos o síntomas. De tal manera que pueden subyacer diferentes síndromes y cuadros clínicos tanto en la etiología como en las complicaciones de los trastornos del aprendizaje y de la conducta. Sin embargo, desde el punto de vista escolar, todos se comportan y rinden en la escuela de manera semejante. Por lo tanto, solo de lo apropiado y oportuno del diagnóstico psicopedagógico dependerá en gran medida la utilización de la estrategia de enseñanza mas apropiada para cada niño, debiendo ajustarse a las necesidades específicas de los alumnos.

El Acta de Individuos con Problemas Educacionales (IDEA, 1990), define al niño con serios problemas emocionales (SED, Serious Emotional Disturbance) expresando que la designación diagnóstica se aplica cuando el niño exhibe una o más de las siguientes características durante un largo período de tiempo, al grado tal que afecte adversamente el rendimiento escolar:

1. Una dificultad o una incapacidad para aprender que no pueda ser explicada por factores intelectuales, sensoriales o de salud física;

2. Una dificultad o una incapacidad para construir o mantener relaciones satisfactorias con sus compañeros y con sus profesores;

3. Conducta inapropiada o respuestas emocionales inapropiadas frente a circunstancias corrientes;

4. Notoria disposición de ánimo de infelicidad;

5. Tendencia a desarrollar síntomas físicos o temores asociados con problemas personales o escolares.

Desde luego, esta definición reúne a un grupo heterogéneo de niños, que pueden clasificarse dentro de distintos sub-grupos en riesgo de bajo rendimiento y/o de fracaso escolar.

La designación de estudiante en riesgo refleja el reconocimiento de que algunos estudiantes están predispuestos a experimentar problemas tanto en el rendimiento en la escuela como en sus vivencias personales y sociales (Johnson, 1997). 
Un estudiante en riesgo no significa que sea retrasado o que tenga alguna incapacidad, sino más bien se refiere a características y circunstancias de su medio escolar, familiar y social que lo predisponen a experiencias negativas tales como deserción, bajo rendimiento, trastornos emocionales, drogadicción, trastornos conductuales, etc.

Los factores de riesgo del estudiante incluyen déficits cognitivos, del lenguaje, atención lábil, déficits en las habilidades sociales y problemas emocionales (Johnson, 1998) y los factores de riesgo presentes en la escuela se refieren a aquellas características y circunstancias específicas ligadas a los docentes y administrativos, la estructura, el clima organizacional y los valores del sistema escolar (Broman, Bien y Shaugenessy, 1985; Rutter, 1980).

La falta de recursos y carencia de estrategias de enseñanza adecuadas, y la violencia escolar, decrecen las posibilidades de éxito personal y académico de los alumnos (Johnson, 1998). Los prejuicios de los profesores, la inhabilidad para modificar el curriculum y las bajas expectativas de rendimiento, coloca a los estudiantes, en riesgo de bajo rendimiento y de fracaso escolar (Ibáñez, 1996; 1998; Taylor y Reeves, 1993). Asimismo, es poco probable que sólo el perfeccionamiento de metodologías de enseñanza pueda disminuir los problemas de rendimiento escolar, si no hay una comunicación eficiente entre la familia y la escuela (Assael y Newmann, 1989).

\section{Familia ansiogénica}

Otra circunstancia ambiental que contribuye al desarrollo y mantención de la ansiedad, proviene del núcleo familiar. La familia está a cargo no sólo del cuidado físico, sino también del bienestar y desarrollo psicológico y social de los hijos. Los niños tienen en la familia sus primeras y más importantes relaciones interpersonales; en el núcleo familiar radican los primeros sentimientos de felicidad o de tristeza per- 
sonal. Allí aprenden un concepto de sí mismos, de los demás y del mundo, que pueden determinar muchas de sus conductas posteriores.

Distintos autores sugieren que los trastornos ansiosos se generan en la familia (Barret, 1998; Last et al., 1991). Relaciones intrafamiliares coercitivas provocan ansiedad en los hijos (Doll y Lyon, 1998). Un ambiente familiar inadecuado, provoca déficits en el desarrollo emocional y genera distintos niveles de ansiedad y de desórdenes ansiosos (Hansen, Sanders, Scott y Last, 1998; Johnson, 1998), especialmente si los padres sufren de ansiedad y/o depresión, y si se producen conflictos y discordias en forma sostenida. Aparentemente se hereda una tendencia general a la reacción ansiosa ante un estímulo (Gray, 1985).

Las experiencias familiares pueden influir en la manera como el niño se percibe a si mismo, su capacidad de control emocional y conductual, y éstas cogniciones pueden contribuir a que se desarrolle y se mantenga la ansiedad (King, Mietz y Ollendik, 1995).

La ansiedad en los padres, es el factor más importante en el desarrollo de la ansiedad en el niño (Cobhan et al., 1998). Niños criados bajo condiciones de abusos físicos y emocionales, tienen mas posibilidades de desarrollar trastornos psicológicos y problemas conductuales. Conflictos maritales y familias disfuncionales son predictores de desajustes emocionales en los hijos. En muchos casos, niños criados en familias conflictivas, tienden a reproducir las mismas condiciones en las familias que ellos forman cuando adultos, perpetuando el ciclo conflictivo. Numerosos estudios muestran que la prevalencia de problemas emocionales y conductuales en la adultez, aumentan en progresión geométrica cuando los niños están expuestos a dos o más factores de riesgo (Doll y Lyon, 1998; Kolvin et al., 1988). Asimismo, la violencia hacia los pequeños es más probable en familias llenas de ansiedades (Barret et al., 1996).

Cuando al menos uno de los padres se caracteriza por ser excesivamente protector y demasiado solícito es un factor que parece 
contribuir al desarrollo de reacciones ansiosas en los hijos. Tal padre puede hacer ver al niño su insuficiencia al restringir y por otra parte controlar todas las actividades no asignándole ninguna responsabilidad y enfatizando las dificultades y los peligros relacionados con la vida (Barrett, 1998). Asimismo, si al menos uno de los padres se caracteriza por ser una persona perfeccionista y exigente, este padre, en general, considera al niño como un adulto pequeño y mantiene expectativas irreales. Bajo estas circunstancias, el hijo aprende rápidamente que es incompetente. Muchos esfuerzos no son suficientes para ganar la aprobación, y trata entonces de ser perfecto. Este niño entonces se vuelve hipercrítico y reacciona excesivamente al fracaso o a errores menores.

Al mismo tiempo, la institución escolar coloca una serie de demandas sobre los alumnos y a través de ellos sobre el núcleo familiar. Los padres se prueban a sí mismos a través de las notas obtenidas por sus hijos; esto muchas veces provoca problemas en la conducta infantil, que traducen una triangulación de los conflictos familiares y de los padres con la escuela (Sarquis, 1993). En general, la humillación originada por pequeños fracasos escolares es transitoria. Sin embargo, algunos niños sienten sus fracasos mas que otros. Llegan a la escuela después de haber sufrido en el hogar mayores fracasos que sus compañeros (Jackson y Frick, 1998).

El apoyo de los padres hacia sus hijos aparece determinado por una valoración de los aprendizajes escolares, como un factor fundamental de desarrollo y por una valoración de la propia capacidad para apoyar este proceso, independientemente del nivel socioeconómico y cultural al que pertenecen (Mc Allister, 1990); asimismo, se asocia a las características de los padres y de los hijos, el contexto familiar y las actitudes de los profesores hacia los padres y hacia los niños (Osborn, 1990; Villalón, De Castro y Streeter, 1998).

La escuela chilena ha ejercido un rol formativo precario, otorgando énfasis a los contenidos mínimos obligatorios y al desarrollo de habilidades cognitivas, descuidando el aspecto emocional-afectivo de los 
Algunas características familiares y de la escuela que contribuyen a la etiología de la tensión

estudiantes, ya que el rendimiento escolar, traducido en calificaciones ha sido considerado como el único indicador de funcionamiento de la escuela.

Un número importante de alumnos ven afectado su rendimiento en las aulas por problemas emocionales, y muy pocos niños y sus familias reciben asesoría en salud mental. Ocurre aún a menudo, que los niños con problemas emocionales asisten a clases interactuando con los demás, incluidos sus profesores en forma maladaptada, lo que exacerba sus ya afectadas relaciones interpersonales y mantiene y hasta amplifica sus problemas emocionales y conductuales, colocándolos en alto riesgo de bajo rendimiento y de fracaso escolar. Con la reforma educacional chilena, se ha prolongado la jornada escolar, y con ello, los alumnos están la mayor parte del día con sus profesores y sus compañeros de curso, lo que aumenta la importancia de las relaciones interpersonales que el niño construya en la escuela para el desarrollo de su autoestima y crecimiento personal.

Dada la evidencia etiológica que implica a las familias de los niños ansiosos, en el desarrollo y mantenimiento de la ansiedad, tiene sentido el incorporar a los padres en intervenciones que alteren las variables familiares que contribuyen en la génesis y desarrollo de esta sintomatología. Especialmente en aquellos casos en que la ansiedad afecta el rendimiento escolar, es imprescindible, además, una relación intersistémica eficiente familia-escuela. Existe una disfunción relacional entre el microsistema escolar y el microsistema familiar, exacerbada por el fomento de la competencia y del individualismo en el sistema escolar. Este problema de relación, es fuente de ansiedad en la familia, especialmente en aquellas cuyo hijo presenta problemas de conducta y de rendimiento en la escuela. El niño ansioso tiene dificultades para lograr su crecimiento y su autoafirmación personal y para desarrollar adecuadamente vínculos con sus compañeros, su integración al grupocurso, su relación con sus profesores y con otras personas. Necesita condiciones en la escuela y en la familia para un desarrollo afectivo sano.

Los aspectos afectivos de los educandos, tales como la identificación y diferenciación de las emociones, sus necesidades, intereses y 
motivaciones, como asimismo sus expresiones emocionales, generalmente se ven minimizadas por la necesidad social de autocontrol y control de los impulsos. Es necesario desarrollar en los estudiantes la capacidad de aprender a inhibir algunas conductas no deseadas y la capacidad de tolerar las frustraciones y límites impuestos por el sistema escolar.

Una manera de ayudar efectivamente a los escolares en estos aspectos está en el desarrollo de los objetivos transversales en la tarea educativa. Los Objetivos Fundamentales Transversales, contenidos en la Reforma Educacional chilena, tienen un carácter orientado al desarrollo personal y a la conducta moral y social de los alumnos y deben perseguirse en las actividades educativas, ya que es ampliamente aceptado que la modernidad ha condicionado nuestras vivencias, afectando el sentido de la solidaridad, de la convivencia y de la participación social, fenómeno que puede revertirse en una escuela que trasmita a sus alumnos estos valores actualmente debilitados. La comprensión del proceso educativo requiere de su estudio como realidad que forma parte del desarrollo integral de un alumno, en un contexto familiar y sociocultural concreto, como proceso de interrelación dinámica del estudiante, con su familia, con sus compañeros y con sus profesores.

\section{Referencias}

Achembach, T.M. (1993). Empirically based taxonomy: How to use syndromes and profiles types derived from the CBCL/4-18,TRF and YSR. Burlington, VT: Department of Psychiatry University of Vermont.

Assael, J. y Neumann, E. (1989). Clima emocional en el aula. Colección etnográfica 2. Santiago de Chile: PIIE.

Barret, P. (1998). Evaluation of Cognitive-Behavioral Groups Tratments for Childhood Anxiety Disorders. Journal of Clinical Child Psychology, 27, 4, 159-468.

Barret, P., Rapee, R., Dadds, M. y Ryan, S. (1996). Family enhancement of cognitive style in anxious and aggresive children. Journal of 
Abnormal Child Psychology, 24, 187-203.

Beidel, D. y Randall, J. (1994). Social phobia. En T. Ollendick, N. King y S. Yule (Eds.), International Handbook of Phobic and Anxiety Disorders in Children and Adolescents (pp. 111-130). Nueva York: Plenum Press.

Bell-Dollan, D. J.( 1995). Social cue interpretation of anxious children. Journal of Clinical Child Psychology, 24, 2-10.

Bender, W. N. (1996). Teaching students with Mild Disabilities. Boston: Allyn and Bacon.

Bermeosolo, J. y Pinto, A. (1996). Caracterización de una muestra de alumnos asistentes a grupos diferenciales en escuelas municipales de tres comunas de Santiago Poniente. Boletín de Investigación Educacional. Pontificia Universidad Católica de Chile, 11, 369393.

Berstein, G. y Borchardt, C. (1991). Anxiety disorders of childhood and adolescence. A critical review. Journal of theAmerican Academy of Child and Adolescent Psychiatry, 30, 519-532.

Broman, S. , Bien, E. Y Shaugenessy (1985). Low achieving children: The first seven years. Hillsdale, NJ: Lawrence.

Cobhan,V., Dadds, M. y Spence, S. (1998). The Role of Parental Anxiety in the Treatment of Childhood Anxiety. Journal of Consulting and Clinical Psychology, 66 (6), 893-905.

da Gloria, J., Pahlavan, F., Duda, D. y Bonnet, P. (1994). Evidence for a motor mechiasm of pain-induced agression instigation in humans. Agressive Behavior, 20, 1-7.

Doll, B. y Lyon, M. (1998). Risk and resilience: Implications for the Delivery of Educational and Mental Health Services in the Schools. School Psychology Review, 27 (3), 348-363.

Gray, J. (1995). Issues in the neuropsychology of anxiety. En H. Tuma y J. Masser (Eds.), Anxiety and the anxiety disorders (254-276). Hillsdale, $\mathrm{Nj}$ : Erlbaum.

Hansen, S., Sanders, S., Scott, M. y Last, C. (1998). Predictors of Severity Absenteeism in Children with Anxiety- Based School Refusal. Journal of Clinical Child Psychology, 27, 3, 246-254.

Ibáñez, N, (1996). La emoción. Punto de partida para el cambio en la 
cultura escolar. Rev. De la Universidad Metropolitana de Ciencias de la Educación 2, 47-58.

Ibáñez, N. (1998). Los profesores y la reforma. Estudios Pedagógicos, 24, 99-106.

Individuals with Disabilities Education Act (IDEA) (1990). October, Public Law 101-476 104 STAT 11³-1151.

Jackson, Y. y Frick, P. (1998). Negative Life Events and the Adjustement of School Age Children: Testing Protective Models. Journal of Clinical Child Psychology, 27, 4, 370-380.

Johnson, G. M. (1997). Resilient at risk students in the inner city. McGill Journal of Education, 32, 35-49.

Johnson, G. M. (1998). Students at risk. School Psychology International, 19, 221-237.

Kashani, J. y Orvaschel, H. (1990). A community study of anxiety in children and adolescents. American Journal of Psychiatry, 147, 313-318.

King, N., Mietz, L. y Ollendick, T. (1995). Psychopathology and cognition in adolescents experiencing severe test anxiety. Journal of Clinical child Psychology, 24, 49-54.

Kolvin, J., Miller, F., Fleeting, M. y Kolvin, P. (1988). Social and parenting factors affecting criminal - offese rates. Findings from the Newcastle Thousand Familiy Study. British Journal of Psychiatry, 152, 80-90.

La Greca, A. M. y Stone, W. L. (1993). Social anxiety scale for childrenrevised. Factor structure and current validity. Journal of Clinical Child Psychology, 22, 17-27.

Last, G., Hersen, M., Kazdin, E. y Orvarschel, H. y Perrin, S. (1991). Anxiety disorders in children and their families. Archives of General Psychiatry, 48, 928-934.

Mc Allister, A. (1990). La participación de los padres y su relación con los logros de los niños. Santiago de Chile: CIDE.

Newcomer, P. (1993). Cómo enseñar a los niños perturbados. México: FEC.

Osborn, A. (1990). Resilient children. A longitudinal study of high achieving socially disadvantaged children. Early Child Development and Care, 62, 23-47. 
Patton, J. R., Blackbourn, J. M. y Fad, K. (1996). Exceptional Individuals in Focus (6 ed.). Englewood Cliffs, NJ: Merril.

Rutter, M. (1980). School influences on children's behavior and development. Pediatrics, 65, 208-220.

Sarquis, C. (1993). Introducción al estudio de la pareja humana. Santiago de Chile: Ed. Universidad Católica de Chile.

Spielberger, C. (1985). Anxiety, cognition and effect. En H. Tuma y J. Maser (Eds.), Anxiety and the anxiety disorders (351-376). Hilsdale NJ: Erlbaum.

Taylor, R. y Reeves, J. (1993). More is better: Raising expectations for student at risk. Middle School Journal, 24, 13-18.

Triandis, H. C., Bontemps, R., Villareal, M., Assai, M. y Lucca, N. (1988). Individualism and collectivism: Cross cultural perspectives on self ingroup relationships. Journal of Personality and Social Psychology. 54, 323-338.

Turner, C., Fenn, M. y Cole, A. (1981). A social psychological analysis of violent behavior. En R. Stuart (Ed.), Violent Behavior (797814). Nueva York: Brunner/Mazel.

Villalón, M. , De Castro, J. y Steeter, B. (1998) El desarrollo de los valores de la verdad y el amor en el ámbito familiar. Boletín de Investigación Educacional. Pontificia Universidad Católica de Chile, $13,24-45$.

Wicks- Nelson, R. e Israel, A. (1997). Psicopatología del niño y del adolescente (3 ed.). Madrid: Prentice Hall.

Zillmann, D. (1994). Cognition excitation interdependenciews in the escalation of anger and angry agression. En M. Potegal y J. F. Knutson (Eds.), The Dynamics of Agression (38-55). Hillsdale, NJ: Erlbaum. 